\title{
Hydroxychloroquine and lupus pregnancy: review of a series of 36 cases
}

\author{
Neil M M Buchanan, Elias Toubi, Munther A Khamashta, Fernanda Lima, Sian Kerslake, \\ Graham R V Hughes
}

\begin{abstract}
Objective-To study maternal and fetal outcome of pregnancy in patients with lupus who were exposed to hydroxychloroquine (HCQ).

Methods-The case records of women $(n=33)$ exposed to HCQ during their pregnancies $(n=36)$ and of 53 control patients from a single lupus pregnancy centre were reviewed to determine lupus activity, obstetric experience, and infant outcome. Results-HCQ was not apparently teratogenic. Lupus activity and obstetric outcome in the two groups were similar.

Conclusion-HCQ continuation is probably safe during pregnancy in patients with lupus, but there is no obvious advantage in commencing treatment.
\end{abstract}

(Ann Rheum Dis 1996; 55: 486-488)

Over the past few decades, convincing evidence has accumulated for the efficacy of antimalarial drugs in the treatment of systemic lupus erythematosus (SLE) and discoid lupus erythematosus (DLE). ${ }^{2}$ Anxiety over their possible teratogenic effects on the developing eye $^{3}$ and in causing chromosomal damage ${ }^{4}$ in the early conceptus have discouraged their administration in pregnancy. However, limited safety data have been building up through anecdotal reports and with the publication of Parke's more extensive experience. ${ }^{5-7}$

It has been our policy in our Lupus Pregnancy Clinic to permit use of hydroxychloroquine (HCQ) to continue, and occasionally to permit usage to commence during gestation. We present here our experience in 36 pregnancies in lupus patients, which comprises the largest recorded series of use of this drug in pregnancy.

Lupus Pregnancy Clinic, St Thomas' Hospital, London, United Kingdom

N M M Buchanan

E Toubi

M A Khamashta

F Lima

$S$ Kerslake

G R V Hughes

Correspondence to: Dr Neil M M Buchanan, Lupus Research Unit,

The Rayne Institute,

St Thomas' Hospital,

London SE1 7EH,

United Kingdom.

Accepted for publication 27 March 1996

\section{Patients and methods}

The authors run a lupus pregnancy clinic that accepts tertiary referrals mainly from London and the surrounding home counties of Southern England. The clinic has existed since 1988 , and its philosophy and operation have been described previously. ${ }^{8}$

One hundred and sixty patients with systemic lupus erythematosus (SLE) or related conditions have attended this clinic during pregnancy; the case records of 33 patients, representing 36 pregnancies, who had taken HCQ were reviewed. The records of a control series of 53 patients who had attended the clinic with lupus pregnancy were drawn randomly from our database and analysed in similar fashion for purposes of comparison.

Information pertaining to the indications for, the dose of, and the duration of exposure to HCQ in pregnancy were recorded and an analysis was made of the age and disease profiles of the patients, and their previous obstetric experience. Clinically assessed activity of the disease during pregnancy was quantified using the Lupus Activity Index and a flare was defined as an increase of 0.26 or more from the minimum score during follow up. ${ }^{9}$ Nephritis was distinguished from preeclampsia by the presence of urinary cellular casts. Prednisolone and azathioprine were used on clinical grounds to control disease activity. The pregnancy outcomes in terms of mode of delivery, gestation age, birth weight, and neonatal health were also recorded.

Pregnancy loss included both spontaneous abortion $(<12$ weeks gestation) and fetal death $(\geq 12$ weeks gestation). Premature birth was considered as a spontaneous or induced termination of pregnancy with a live birth between 21 and 37 weeks; termination of pregnancy was defined as a voluntary induced abortion.

\section{STATISTICAL ANALYSIS}

Comparison between proportions was by $\chi^{2}$ analysis. Means were compared using Student's $t$ test for normally distributed variables. Values of $p$ less than 0.05 were considered significant.

\section{Results}

Demographic data of the patients and controls in relation to age and race, mean duration of lupus, antibody pattern, average prednisolone dose, and disease activity at the onset of pregnancy were comparable. The pattern of previous obstetric experience was similar: $42 \%$ of the HCQ group had live births, compared with $36 \%$ of the controls.

Of the 36 pregnancies in 33 patients with HCQ exposure that were reviewed, 22 were exposed to HCQ $200 \mathrm{mg} /$ day and 14 to 400 $\mathrm{mg} /$ day at some point during gestation. The figure illustrates the profiles of exposure.

The mean duration of exposure to HCQ in pregnancy was 28.4 (SD 10.75) weeks, and the average antenatal exposure was $24.4(20.08)$ months. Original indications for the drug were cutaneous rash in every case, with additional arthritis in 18 and serositis in one patient. 


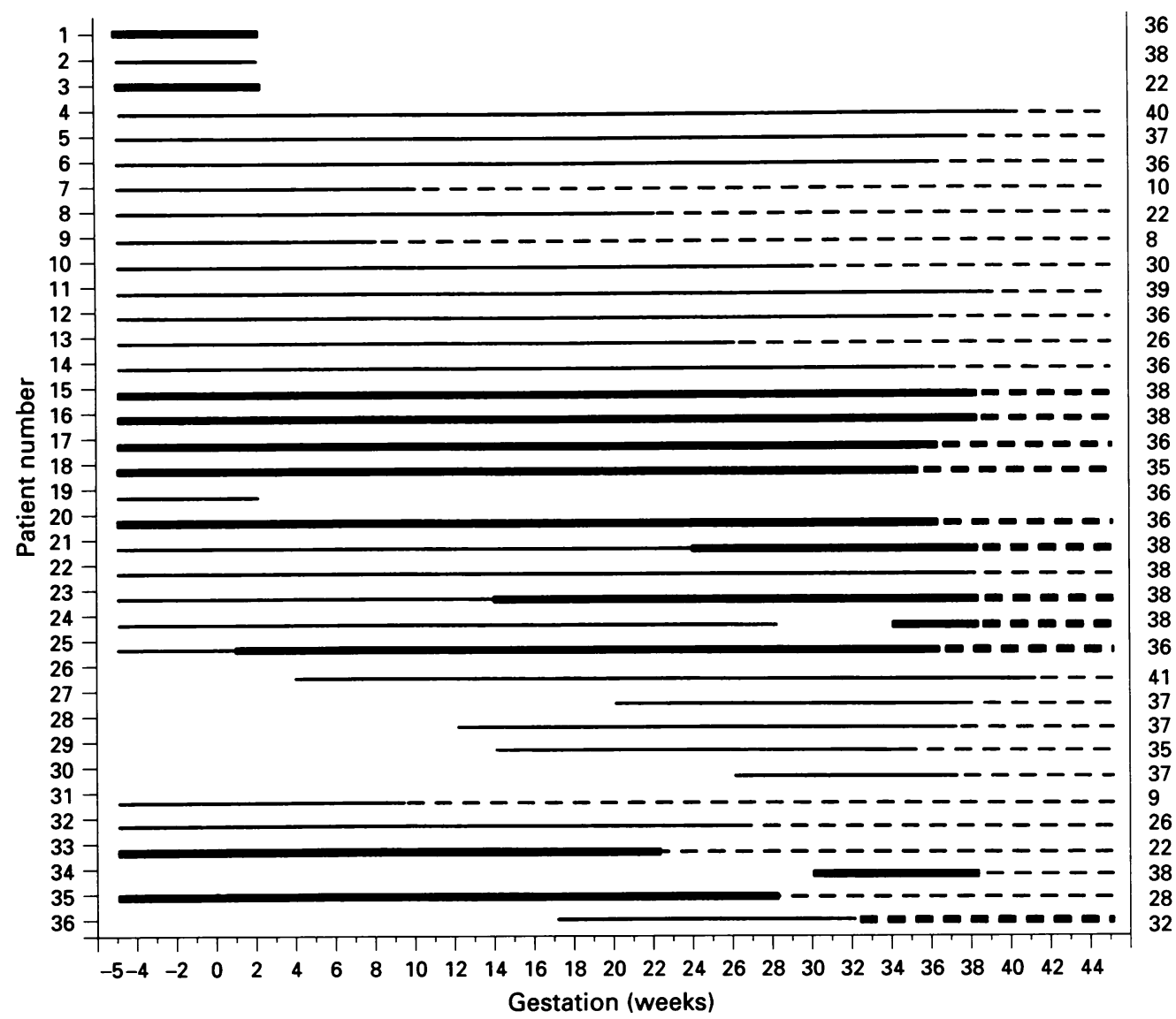

Profiles of exposure to hydroxychloroquine during pregnancy. $-=400 \mathrm{mg} \mathrm{HCQ;}-=200 \mathrm{mg} \mathrm{HCQ} ;---=$ drug continued afier completion of gestation.

Mean duration of antenatal disease from the time of diagnosis was 6.45 (3.97) years; two patients had DLE and the remaining 31 had SLE, according to the classification criteria of the American College of Rheumatology. ${ }^{10}$

Eighty six percent of HCQ pregnancies (31 pregnancies with 32 babies) and $83 \%$ of the control pregnancies (44 pregnancies and 44 babies) ended successfully with a live birth (NS). There were two cases $(6 \%)$ of spontaneous abortion and three $(8 \%)$ of fetal death in the HCQ group. In the control group, there were four cases of spontaneous abortion $(7 \cdot 5 \%)$ and five cases of fetal death $(9 \cdot 5 \%)$.

Prematurity occurred in 17 of the 31 successful HCQ pregnancies (55\%) and in 21 of 44 control pregnancies $(48 \%)$. No statistically significant difference was seen. Table 1 compares other obstetric parameters such as fetal distress, intrauterine growth retardation, induction of delivery, and mean weight of the live birth babies, for both groups. Two cases of neonatal death were observed in the HCQ group and one in the control group; all were related to extreme prematurity.

Table 1 Obstetric outcome in successful pregnancies

\begin{tabular}{lcc}
\hline & $\begin{array}{l}\text { HCQ group } \\
(n=31)\end{array}$ & $\begin{array}{l}\text { Control group } \\
(n=44)\end{array}$ \\
\hline Prematurity & $17(55)$ & $21(48)$ \\
Weight at birth (g) $\dagger$ & $2443 \cdot 71(997 \cdot 35)$ & $2473 \cdot 07(965 \cdot 71)$ \\
Induction of delivery & $19(61)$ & $26(59)$ \\
IUGR & $6(19)$ & $18(41)$ \\
Fetal distress & $10(32)$ & $16(36)$ \\
\hline
\end{tabular}

Values are number (\%) or tmean (SD).

IUGR = Intrauterine growth retardation.

No statistically significant differences between groups.
None of the babies delivered of mothers taking HCQ had congenital malformations, though one baby had Down's Syndrome (born to a 32 year old mother). In the control group, one baby had an extra (sixth) finger in his left hand.

No evidence of visual disturbance was observed clinically in these babies.

When we analysed the antiphospholipid antibody status (anticardiolipin antibody, lupus anticoagulant, or both) of women experiencing fetal loss during either HCQ or control pregnancies, we found that four of five pregnancy losses in the HCQ group $(80 \%)$ and six of nine losses in the control group (67\%) were positive for antiphospholipid.

\section{MATERNAL OUTCOME}

One patient with DLE experienced disease activity at the onset of pregnancy (eight weeks of gestation). Her disease became quiescent and remained so after the introduction of HCQ. A second patient with DLE developed a skin flare at 34 weeks of gestation; this was controlled with an increase in the dose of HCQ from $200 \mathrm{mg} /$ day to $400 \mathrm{mg} /$ day. Table 2 summarises the SLE data (34 pregnancies) relating to disease activity during pregnancy in the HCQ and control groups.

In order to analyse the steroid sparing effect of $\mathrm{HCQ}$, we compared the maximum mean dose of prednisolone received during pregnancy by patients in both the HCQ and the control groups. The means were 13.84 (14.29) $\mathrm{mg}$ and $16 \cdot 13(13 \cdot 43) \mathrm{mg}$, respectively (NS). 
Table 2 Maternal outcome in systemic lupus erythematosus pregnancies

\begin{tabular}{lcc}
\hline & $\begin{array}{l}\text { HCQ group } \\
(n=34)\end{array}$ & $\begin{array}{l}\text { Control group } \\
(n=53)\end{array}$ \\
\hline Total number of flares & $21(62)$ & $31(58)$ \\
Renal flare only & $4(12)$ & $6(11)$ \\
Hypertension & $8(24)$ & $20(38)$ \\
Pre-eclampsia & $1(3)$ & $20(38)$ \\
Azathioprine use & $7(21)$ & $7(13)$ \\
Thrombosis & $1(3)$ & $2(4)$ \\
\hline
\end{tabular}

Values are number (\%).

During pregnancy and the puerperium.

No statistically significant differences between groups.

\section{Discussion}

HCQ is a valuable and possibly underused agent in the treatment of moderate SLE. Our findings support the preliminary evidence for the safety of the drug in pregnancy that was suggested by Parke. ${ }^{6}$ HCQ has been used in pregnancy for malarial prophylaxis with no teratogenic effects, albeit in a considerably lower dosage (400 mg weekly). ${ }^{11}$

With continued use, HCQ accumulates in human tissue, especially the liver, and on discontinuation of the drug the body stores are gradually depleted, with a half life of eight weeks. ${ }^{3}$ Because of this, unless a lupus pregnancy is planned well in advance (two thirds of our series were not), fetal exposure during the critical first trimester cannot be avoided by discontinuing the drug when pregnancy is recognised.

An increased rate of flare is recognised in lupus patients discontinuing $\mathrm{HCQ},{ }^{12}$ and this indeed occurred in the five patients in our series who stopped taking it. Flare is considered undesirable in pregnancy, being related to prematurity and requiring administration of other medication. ${ }^{13} \mathrm{HCQ}$ is claimed to have significant steroid sparing properties in lupus ${ }^{14}$ and we therefore expected that steroid use and dosage might be decreased in our series, but this was not so when compared with the control group. HCQ also has anticoagulant effects and has been used in prophylaxis against deep vein thrombosis, ${ }^{15}$ but the numbers in our series were too small to allow us to support or refute this application.

Four of $14 \mathrm{HCQ}$ patients positive for antiphospholipid had pregnancy failure, compared with six of 24 antiphospholipid positive controls. There was no difference in incidence of maternal thrombosis between the HCQ and control patients.
There are theoretical reasons, therefore, for maintaining HCQ in SLE pregnancy, and 24 of our patients continued to take the drug which had been previously commenced. Seven of those in our series initiated treatment in pregnancy; there were no ill effects observed in mothers or babies in this subgroup. Overall, our experience in a relatively small series of patients demonstrates that continuing HCQ appears to be safe in lupus pregnancy, though there is no obvious advantage in initiating treatment with the drug. It is possible, in view of the retrospective nature of the study, that the number of early miscarriages was underestimated. This may be of importance, as HCQ has been shown to induce chromosomal abnormalities. In addition, accurate assessment of vision in young children is difficult to undertake, and we intend to follow up the babies of patients exposed to HCQ 'in utero' in the future.

1 Ruzicka T, Sommerburg C, Goerz G, Kind P, Mensing H. Treatment of cutaneous lupus erythematosus with
acitetin and hydroxychloroquine. Br $\mathcal{f}$ Dermatol 1992; 127: $513-8$.

2 Rudniki R D, Gresham G E, Rothfield N F. The efficacy of antimalarials in systemic lupus erythematous. $\mathcal{F}$ Rheumatol 1975; 2: 323-30.

3 Ulberg S, Linquist N G, Sjostrand S E. Accumulation of chorioretinotoxic drugs in the fetal eye. Nature 1970; 227: 1257

4 Neill W A, Panayi G S, Duthie J J R, Prescott J R. Action of chloroquine phosphate in rheumatoid arthritis. II. Chromosome damaging effect. Ann Rheum Dis 1973; 32: 547-50.

5 Parke A L. Antimalarial drugs, systemic lupus erythematosus and primalarial drugs, systemic lupus erythe-

6 Parke A, Fox $R$ I, Rynes R I. Antimalarial drugs and pregnancy. $A m \mathcal{F}$ Med 1988; 85: 30-3.

7 Parke A. Antimalarial drugs and pregnancy. Br $f$ Clin Pract 1987; 41: 56-8.

8 Buchanan N M M, Khamashta M A, Morton K E, Kerslake S, Bagualey E A, Hughes G R V. A study of 100 high risk lupus pregnancies. Am $\mathcal{F}$ Rep Immunol 1992; 28: 192-4.

9 Ruiz-Irastorza G, Lima F, Alves J, et al. Increased rate of lupus flare during pregnancy and the puerperium. $\mathrm{Br} \mathcal{F}$ Rheumatol 1996; 35: 133-8.

10 Tan E M, Cohen A S, Fries J F, Masi A T, McShane D J, Rothfield N F. The 1982 revised criteria for the classification of systemic lupus erythematosus. Arthritis classification of systemic

11 Lewis R, Laversen N H, Birnbaum S. Malaria associated with pregnancy. Obstet Gynaecol 1973; 42: 696-700.

12 The Canadian Hydroxychloroquine Study Group. A randomised study of the effect of withdrawing hydroxychloroquine sulfate in systemic lupus erythematosus. $N$ Engl F Med 1991; 324: 150-4.

13 Buchanan N M, Khamashta M, Kerslake S, Hunt B J, Hughes G R V. Practical management of pregnancy in systemic lupus erythematosus. Fetal Mat Med Rev 1993; 5: $223-30$

14 Bell C L. Hydroxychloroquine sulfate in rheumatoid arthritis: long term response rate and predictive arthritis: long term response rate

15 Chrisman O D, Snook G A, Wilson T C, Short J Y Prevention of venous thromboembolism by adminisPration of hydroxychloroquine: a preliminary report. $\mathcal{F}$ tration of hydroxychloroquine: a
Bone foint Surg 1976; 58: 918-20. 